\title{
On the Challenges Facing the Handling of Solid Biomass Feedstocks
}

\author{
D. Ilic ${ }^{1}$, K. Williams ${ }^{1}$, R. Farnish ${ }^{2}$, E. Webb ${ }^{3}$ and G. Liu ${ }^{4}$ \\ ${ }^{1}$ The University of Newcastle, Australia, ${ }^{2}$ The University of Greenwich, U.K., \\ ${ }^{3}$ National Oak Ridge Laboratory, U.S.A and ${ }^{4}$ DuPont, U.S.A
}

\begin{abstract}
With major global emphasis on the management of waste, alternative resources and a shift to advanced, environmentally sustainable technologies, demand for large volumes of heterogeneous solid biomass feedstocks for use in energy, heating and as a biofuel or biochemical is expected to significantly rise. In transforming sporadic supply of a low value, highly variable product, to continuous and controlled high throughput systems, a thorough understanding of the feedstock physical properties will increase in importance. Appropriate characterisation tests are necessary to define technical specification and selection criteria for handling equipment and to adequately appraise the requirement and location for additional processes or pre-treatment (additional energy and cost) to be integrated into the handling chain. Such tests may also be of influence on the material characteristics to be used in the process of converting the feedstock to energy or fuel. This paper discusses the main parameters and the approach to obtain them associated with a number of handling phases. A holistic approach to the design of biomass feedstock handling systems for further development and practical implementation is also proposed.
\end{abstract}

\section{Introduction}

The utilisation of waste is becoming a significant global issue. Landfill space has long been exhausted and poor disposal practices including burning result in detrimental effects such as green house gas emissions and soil contamination. Concurrently, high use of traditional resources required for energy and transportation and reliance on exhaustible fuels have increased demand for renewable and sustainable solutions. Never before observed global climate patterns, air quality and acceptance of environmental degradation directly impacting quality of life have lead to increased governmental policy aimed at incentivising a transition to more environmentally focused technologies and processes. This also includes a significant shift towards second-generation biofuel or feedstock supplies that do not compete with arable land or potentially vital food resources.

Much emphasis is placed on the technologies and process used to obtain energy and quality biofuel from feedstocks, as well as integration of the biomass/renewables into existing energy and fuel supply chains. In order to facilitate competitiveness through the valorisation of biomass, effective application and integration of equipment, technology and systems as well as optimising processes within the handling chain is essential. This will provide improved integration, increased system efficiency and reduce handling chain costs, making the process financially viable.

It has been recognised that agricultural and forestry waste, by-products and residues will alone not be sufficient to meet the level of demand necessary and dedicated sustainable production systems will be required. There is also recognition that agriculture and forestry biomass feedstock production will need to significantly increase in order to meet demand and requirements of a commercial scale bioenergy and biofuel sectors.

In developing countries, lack of access to affordable, reliable, safe and environmentally sustainable energy sources has seen an increase in the development of biogas applications (anaerobic digesters). Such developments also may be used for secondary benefits such as natural fertiliser as a by-product. 
Globally, in 2016, renewable energy sources in power generation have reached $2.8 \%$ of global energy consumption. This is significantly lower compared to oil (32.9\%), coal (39.2\%) and gas $(23.8 \%)$ [BP Statistical Review of World Energy]. However, according to the same report, renewable energy consumed in power generation grew by $15.2 \%$, which is approximately equal to the entire increase globally. Renewable energy consumption is highest in Europe and Eurasia (39.2\%), followed by Asia Pacific (30.4\%) and then North America (22.6\%). Similarly, the U.S. Energy Information Administration, International Energy Outlook 2016 cites renewables as the worlds fastest-growing energy source, with consumption estimated to increase by an average of $2.6 \%$ per year from 2012 to 2040 [USEIA].

Through increased government incentives, according to the International Renewable Energy Agency (IRENA), renewable energy could account for up to $36 \%$ of the global energy mix by 2030 with biomass accounting for up to $60 \%$ [IRENA]. This is perhaps offset by generally high costs associated with set-up and operation for plants using the latest conversion technologies. Such biomass statistics, and many more referenced in a variety of current research, from a sustainability perspective, have the potential to impact demand of other commodities.

Justification in shifting to a renewable energy technology may satisfy moral and environmental requirements, significant barriers are also evident in transforming from pilot scale to commercialisation (termed pioneer plants in North America). For example, a proven and effective, second generation bio-ethanol pilot plant, unavoidably reaches an obstacle that requires an influential, well established supporting organisation, willing to take on existing federal and/or state legislation. Such an ordeal in turn is related to lack of purpose built infrastructure for on-time, cost effective, efficient, continuous and reliable supply of high throughput, high density feedstocks. This journey is also heavily intertwined with societal dependence on traditional, well established fossil fuels and resources.

Previous studies into the research necessities of biomass characterisation have been presented by [Ramirez-Gomez]. That work outlined a number of areas requiring further research related to understanding biomass behaviour during storage, flow and handling including methods for measuring PSD, flowability and strength, durability, self-ignition/oxidation and explosibility. Such parameters effectively drive equipment and plant layout design, as well as selection of most appropriate mode of conveying, transportation, storage and feeding. This paper looks to further elaborate on these aspects and proposes a framework to facilitate the transition to a higher volume system.

While characterisation is necessary to define the materials handling equipment and processes, characteristics of the feedstock are effectively defined by the conversion process requirements with respect to producing highest energy or sugar yield. According to [Sharma et al], biofuel refineries may be classified into four types: starch, sugar, oil and lignocellulosic based. Energy from feedstocks is utilised by direct combustion or converting to solid, gas or liquid with main conversion technologies including thermo-chemical processes (pyrolysis and gasification), bio-chemical processes (anaerobic digestion and fermentation) and physicochemical processes. In addition to this, depending on feedstock characteristics, development of cost-effective pre-treatment processes may also be required for conversion. Taking these aspects into consideration, it is important to note that material feed and handling systems have been cited as the most common causes of downtime in thermochemical conversion processing [Craven et al].

A shift towards reducing environmental footprint and sustainability has resulted in utilisation of advanced second-generation biofuels from lignocellulosic materials (cellulose, hemi-cellulose and lignin) utilising products that do not compete with land for growing food crops. This is in contrast to first-generation commercial cellulosic feedstock sources and processes, primarily at the pioneer scale. 
In North America, corn stover biomass is the primary feedstock of choice for first-generation cellulosic bio refineries [Shah, Darr]. Lignocellulosic feedstock sources include agricultural residue, herbaceous crops, invasive weeds, short rotation woody crops, urban woody waste, sawmill residue and forestry biomass [Sharma et al].

With respect to handling aspects, the biomass feedstock supply chain consists of storage, feed (loading/unloading) and transportation, all requiring appropriate and favourable principles for design in order to maintain the low emission benefit of using biomass. To provide a means of effectively designing systems to handle the heterogeneous biomass feedstocks, existing procedures to obtain representative samples, prior to the characterisation process, will need to be reviewed. Representative sampling represents the first crucial step in accurate characterisation test work.

Physical characterisation is then required, which will become the basis to drive equipment and facility specification criteria and selection based on the properties of the handled individual feedstocks, in view of the operational requirements for a economically viable conversion process. Characterisation would also form the basis for evaluating if processing or pre-treatment (ease of handling) and conditioning (influencing specific attributes) is required and at which stage of the supply chain is most optimal - in order to facilitate handling storage and transportation of the biomass material. This is a vital criteria that also will influence the location and the delegation of responsibility of the processing/treatment, ultimately also directly impacting financial considerations included in the business development model of the enterprise and required to necessitate trade of the valuable commodity. In turn, such information will also decide the location where the feedstock should be processed, who should process it (producer or user), in which form it is to be supplied in and which pre-defined characteristics must be met. Characterisation will also allow determining if integration or application of existing equipment and facilities available is not possible. The feedstock characteristics such as particle size for example, may then be cross-correlated to those specifically required by the conversion process technology (i.e. at the bio refinery).

Due to the extremely low energy density per unit mass of raw biomass, pre-treatment of the feedstock is necessary. Some of the different approaches include torrefaction, carbonization, pelletizing, chopping, shredding and grinding [Ramirez-Gomez]. Densification or increasing the density of the handled product is also required to reduce transportation costs by allowing transportation of larger quantities of bulk solids and trade more economically feasible. It is important to note that the process used to transform raw biomass feedstocks into more handleable bulk solids needs to be taken into account when assessing the overall carbon footprint (life cycle analysis) and possible impact on the environment in utilising the feedstock end-product. This for example also includes other factors required for their modification including type of binder used [Kaliyan]. Globally, wood pellet production and demand has significantly increased over the previous five years. This has eventuated through incentives driven by European Commission's 2020 plan for reducing greenhouse gas emissions and increasing renewable energy percentage of total energy generation. Drax power station in the U.K., imports wood pellets from North America and Canada, contributing to the U.K. being the world's largest importer of wood pellets. The U.S. is the largest exporter of wood pellets globally with exports increasing almost 40\% (2013-2014). In 2014, around three quarters of these exports were supplied to the U.K, with Drax power plant accounting for over $80 \%$ [USIEA].

\section{Storage, Handling and Transportation}

In a recent paper on cornstover feedstock supply in the Midwestern U.S., [Shah, Darr] estimate that fuel and labour amount to around $50 \%$ and $60 \%$ of the total cost of feedstock transportation (based on trucking) respectively. One critical aspect of biomass valorisation is reduction of transportation 
costs through the development of efficient logistic systems. This includes reducing capital costs through utilisation of existing logistics for feedstock transportation. Considering the generally low density per unit mass of biomass feedstocks, long distance transportation is not economically feasible unless efficient handling transportation and storage systems are implemented.

A number of researchers have commenced with assessing biomass feedstocks by relating them to traditionalist flow parameters and either using well established characterisation equipment or defining new characterisation equipment based on what is most appropriate for the tested feedstock. Undoubtedly, the procedure envisioned must involve a performance comparison to some existing resource such as coal for example (or other well utilised bulk solid) as a means of establishing limitations and applicability of traditional tests and procedures, prior to verifying the suitability of a design or equipment required for transportation and handling purposes. For example, in discussing a dedicated dry handling bulk terminal facility [Wu et al], tested feedstocks including wood pellets, chips and torrefied pellets. The programme involved assessment of particle size distribution (PSD), internal angle of friction and degradation. Similarly, [P.S. Lam] has identified engineering properties of biomass based on application and lists density and angle of repose as design parameters for handling, storage and transportation as well as discussing characterisation methods for their determination. Specification of the applicable test and most appropriate procedure to classify and assess feedstock physical properties including particle characterisation, degradation and moisture effects is also required.

Investigation into application of fundamental principles associated with traditional flow properties and arching behaviour has previously been studied by [Miccio et al, Wu et al, Hinterreiter and Khan]. [Miccio et al] indicate that, shear cells could be used to assess flow properties if particle size is below $2 \mathrm{~mm}$ (for the biomass solids tested). Importantly, that work also noted particle elasticity, shape and internal shear as properties that influence flow towards unexpected behaviour, generally related to achieving steady state during compaction (consolidation).

It appears evident that feedstock bulk density will be one of the most critical characterising parameters of influence on the economics of biomass trade. Symbolically, [Shah, Darr] cite feedstock (in this case bale) density as being of most influence on the cost to deliver stover to the bio refinery. Of particular significance, due to elasticity, research into compacted storage beyond traditional stress analyses is required, where focus needs to be measurement of tensile force due to the bulk solids "springiness". Such test work will need to quantify un-restrained, un-consolidated dilation, which goes beyond current compressibility assessment methods. This may be obtained through development of a test apparatus to establish the relationship between compaction, dilation and the applied stress.

Such a relationship is demonstrated on granulated wattle and wheat straw in Figure 1 to Figure 3 . The test was conducted in a $63.5 \mathrm{~mm}$ diameter, $19 \mathrm{~mm}$ deep cell, which is typically used for mined material assessment of the finer sized fractions (typically less than $5 \mathrm{~mm}$ ). For the wheat straw, the sample was first cut into small pieces in the order of $5 \mathrm{~mm}$ to fit in the cell in a loose poured state. Loads were applied by a means of a lid and weight carrier, and the consolidation/dilation of the sample measured via a linear variable displacement transducer (LVDT). Knowing the sample volume, mass and applied loads allows for the consolidation/dilation relationship to the normal consolidation pressure to be determined. 

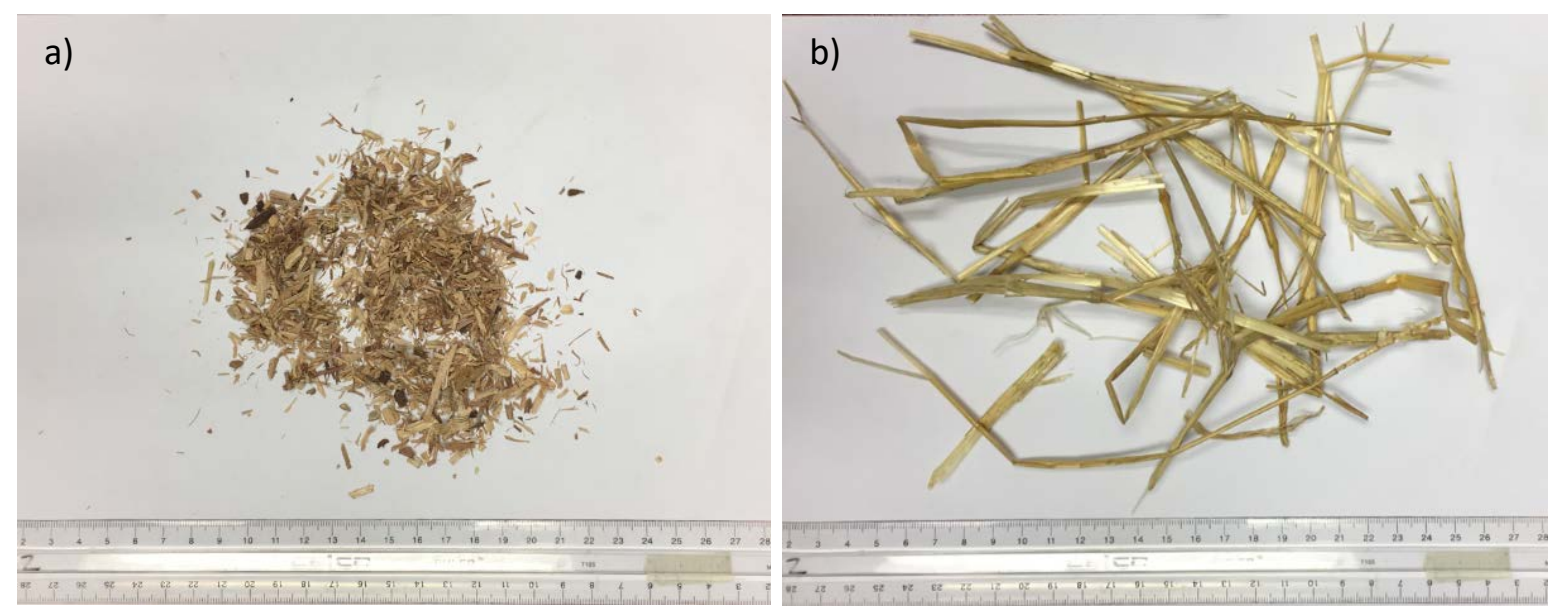

Figure 1: a) Granulated Wattle and b) Wheat Straw
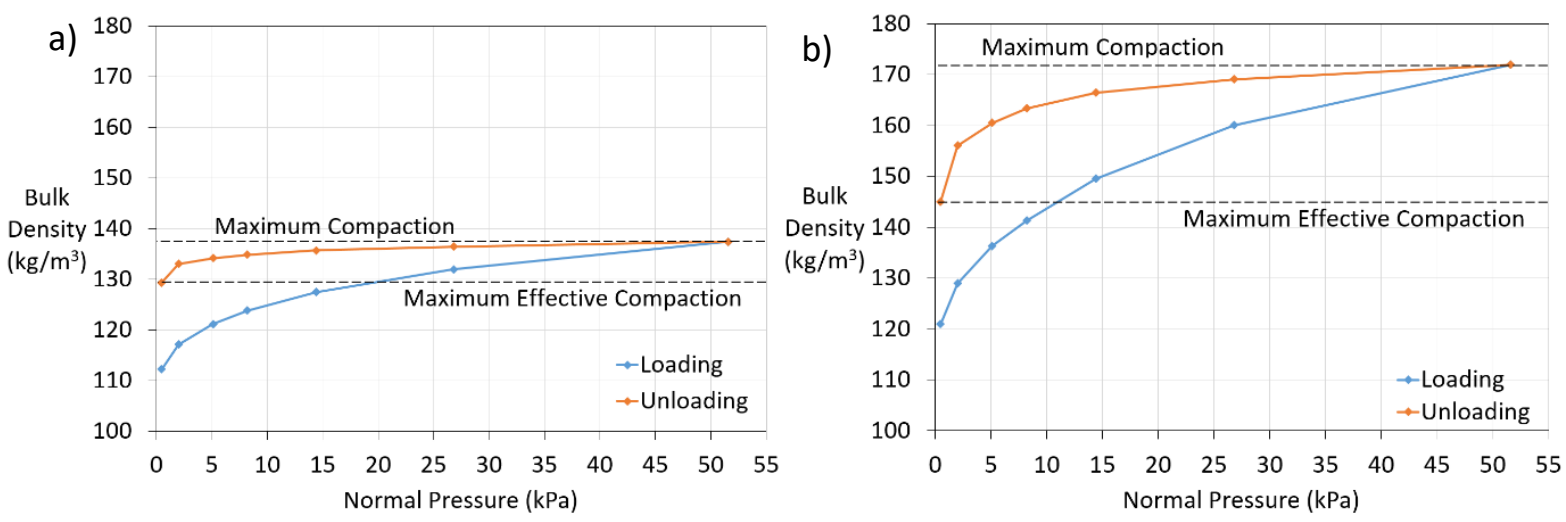

Figure 2: Consolidation/Dilation response of (a) Granulated Wattle and b) Wheat Straw
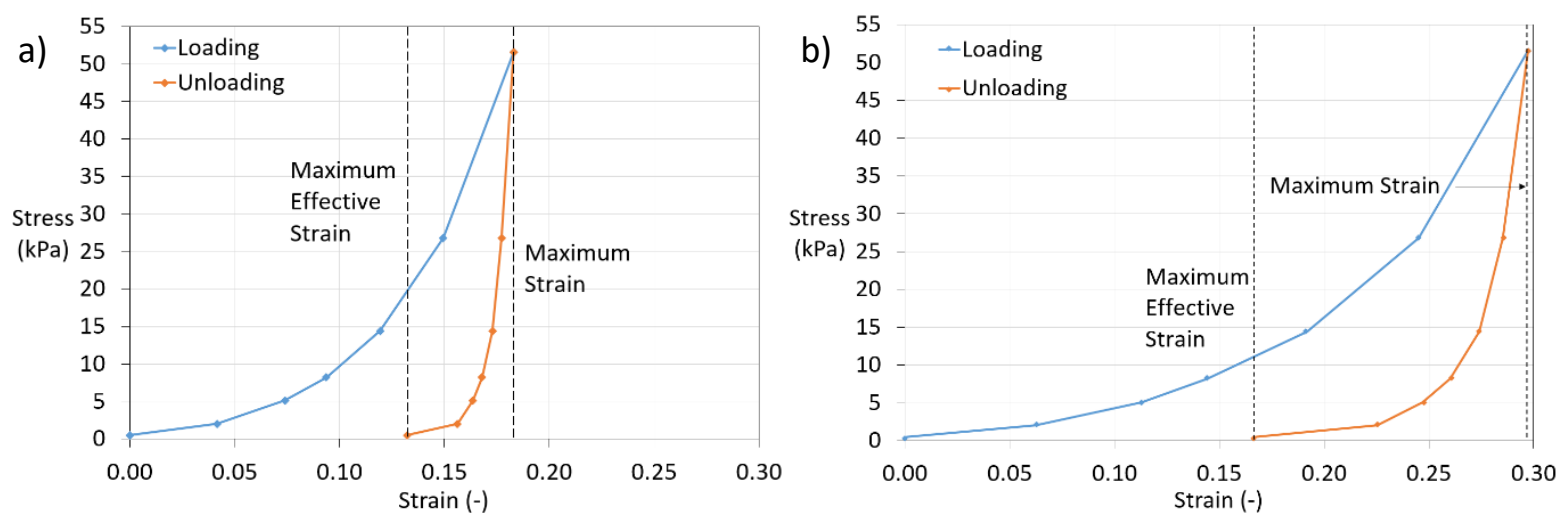

Figure 3: Corresponding Stress/Strain response of (a) Granulated Wattle and b) Wheat Straw

The data from the compaction/dilation test allows for a hysteresis relationship of the material to be established: consisting of the total energy to compress, plastic and elastic energy response. In the above case, the plastic energy response to compression, the area between the two curves, for granulated wattle and wheat straw, was calculated to be $2.0 \mathrm{~kJ} / \mathrm{m}^{3}$ and $3.0 \mathrm{~kJ} / \mathrm{m}^{3}$. The corresponding maximum compaction (compressibility) observed is approximately $22 \%$ and $42 \%$ respectively. However, upon removal of consolidation, the materials dilate, resulting in a compaction loss in the order of $6-19 \%$ respectively. This means that the maximum effective compaction, for the applied load tested, is $15 \%$ for the granulated wattle and $20 \%$ for the wheat straw. An alternative relationship describing the ability of the feedstock to deform under load can also be observed through the strain response to normal pressure (stress) plots presented in Figure 3 . These results demonstrate greater 
benefit in applying a further size reduction process (as a means of densification) to the wheat straw. Note that particle density of granulated wattle and wheat grass was measured (via a nitrogen displacement pycnometer) to be $1396 \mathrm{~kg} / \mathrm{m}^{3}$ and $1596 \mathrm{~kg} / \mathrm{m}^{3}$ respectively. The results are summarised in Table 1. Together with the stress/strain relationship depicted in Figure $3 \mathrm{~b}$ ), the results show inefficiency in compacting the wheat straw in the state tested.

Table 1: Compaction Efficiency

\begin{tabular}{|c|c|c|c|c|}
\hline Feedstock & $\begin{array}{c}\text { Particle Density } \\
\left(\mathbf{k g} / \mathbf{m}^{\mathbf{3}} \mathbf{)}\right.\end{array}$ & $\begin{array}{c}\text { Maximum } \\
\text { Effective }_{\text {Compaction }^{\mathbf{1}} \mathbf{( \% )}}\end{array}$ & $\begin{array}{c}\text { Compaction } \\
\text { Efficiency }^{\mathbf{2}} \mathbf{( \% )}\end{array}$ & $\begin{array}{c}\text { Maximum } \\
\text { Effective Strain } \\
\text { (\%) }\end{array}$ \\
\hline Granulated wattle & 1396 & 22.4 & 9.3 & 13 \\
\hline Wheat straw & 1596 & 42.2 & 9.1 & 17 \\
\hline
\end{tabular}

Note: ${ }^{1}$ Maximum effective compaction = maximum unloaded bulk density/initial unconsolidated bulk density ${ }^{2}$ Compaction efficiency $=$ maximum effective compaction/particle density

\section{Handling Issues}

Although the use of biomass as an energy source has great potential, in some cases the challenges for the design of efficient plant are under estimated. The key cause of many problems is a lack of meaningful bulk characterisation at the earliest stages of plant or equipment design. Depending upon the process, biomass input stock can take an engineered form (pellets), nominally regular particles (virgin wood chip) or highly irregular particles (forestry residue). Each of these generic fuel forms has its own design requirements and requirements which should dictate the form and layout of plant. Parameters that can be assessed for practical design purposes include particle hardness, which has direct implications for the generation of fugitive material through the process, plant wear rates and compaction efficiency.

Particle breakage and dust generation is another concern for biomass handling. Taking pelletised fuel as an example, impact of the pellets in chutes and transfer points is a major cause of breakage and dust evolution. Failure to understand the level of dust generation can have several detrimental effects ranging from blinding of dust filtration systems that are undersized, to changes in the bulk flow behaviour of the pellets. The latter issue is far from unusual, with plant being designed on the basis of handling 'clean' pellets with a nominal value of 'fines' content. The reality in many poorly designed plants, the fuel specification (in terms of bulk characteristics) is far removed from the design basis with higher than anticipated fines content and a consequent increased severity of segregation in storage equipment (which will concentrate the fines that are present). From experience, specifications for $5-8 \%$ by weight sizing less than $3.15 \mathrm{~mm}$ often equate to up to $25 \%$ by weight. Clearly, what may present an easy to handle, free-flowing material can be quite a different material towards the end of the process. Increasing fines content will result in decreased flowability - with the consequence of a higher incidence of irregular flow or no flow at all from storage bunkers or silos.

Chipped, forestry derived, or architectural reclaim fuels present a different range of potential problems. Not least of the problems is how to characterise the bulk materials for the purposes of specifications and design. The main challenge lies in the fact that the particles are irregular in shape and often dominated by elongated or flat particle forms - along with secondary, abrasive/corrosive contaminants and possibly uncontrolled moisture content, depending up storage conditions prior to dispatch to the end user. The irregular nature of the particle shapes that comprise the bulk rule out the adoption of conventional approaches for flow characterisation that are predominantly used for granular or powder bulk forms. A particular issue with the bulk storage behaviour lies in the bulk elasticity exhibited by the less refined biomass forms. This compressibility (in contrast to 'clean' pellets) endows these fuels with the capability to form flow stoppages or 'hang-ups' (arches) over far 
greater outlets than could be implied by a cursory inspection of the fuels. At the simplest level of scrutiny, such fuel types appear to offer free-flowing behaviour - if compressed by hand, no strength is retained. However gains in strength are substantial (with minimal lateral load transmission) if increased vertical loads are applied to a bed of material. This behaviour effectively rules out the use of storage equipment that employs discharge through a convergent vessel section (i.e. a hopper type arrangement). This dictates that vertical or dilatant walled storage schemes may be necessary where a metered, predictable and/or controlled outfeed is required by the process. The consequence for the adoption of this type of design is that a full live discharge arrangement is required if the generation of non-flowing regions (and the risk of biologically driven self-heating) are to be avoided. This approach adds significantly to installation costs and is often an area of engineering where 'short cuts' are applied to fit the project into the capital expenditure (CAPEX) budget. The resulting poor design and/or underperformance of the system greatly impacts on process reliability and overall plant availability.

The importance of obtaining an early understanding of the fundamental bulk characteristics of current and future sources of fuel should be the foundation for the design of plant that has the capability to operate at its intended output over its life cycle. A consideration of market pressures and multi fuel; source strategies makes this prerequisite a critical factor in designing for plant optimisation, maximising efficiency of process and thereby minimising risk.

\section{Characterisation for Design and Numerical Modelling}

Following the groundbreaking work of Jenike [Jenike], which generally involved performing flow property testing on the fine content (say below $4 \mathrm{~mm}$ ) of a bulk solid material sample, traditional continuum mechanics based approaches have been the basis for design of bulk solid materials handling systems. While fundamental association between physical properties and bulk solid behaviour developed from that work, such as cohesive arching and the internal angle of friction, remains a trend, which has significantly grown over the last decade, has seen systems designed via the use of advanced and detailed interaction-modelling packages. Generally, these approaches include evaluation of:

- Inter-particle interactions and particle interactions with the surrounding boundaries [Discrete Element Modelling (DEM)],

- Interactions between particles, boundaries and the surrounding air [Continuum Fluid Dynamics (CFD) or coupled DEM/CFD] or liquid [Smoothed Particle Hydrodynamics (SPH)]

- Resultant effect that bulk behaviour transmits to surrounding structures [Finite Element Analysis (FEA) or coupling FEA/DEM].

In recent times, research and simulation has also extended to coupling DEM with heavy equipment modelling packages for example such as Multi Body Dynamics (MBD). From these tools, DEM is arguably the world leader in implementation and applicability to the design and modelling the handling of particulate bulk solid handling systems, in turn-around times acceptable to industry demands. In this type of simulation, rigid particles are modelled with bulk deformations accounted for in the contacts between them.

Calibration of modelling parameters for use with DEM has been rather well documented [Groger, Johnstone, Grima]. Due to the disparity between physical properties obtained via traditional tests (e.g. the Jenike direct shear tester) and simulation parameters, such as bulk loading stiffness (spring force at particle contacts), sliding and rolling friction, so termed calibration tests become essential. These tests are typically conducted on full size bulk solid samples in bench-scale laboratory apparatus and then comparison to traditional flow property tests is undertaken. Such an interface, between physical macro parameters and those used in the simulation model (microscopic), is via an iterative modelling 
procedure through which simulated bulk behaviour is examined until it reflects bulk behaviour as would be expected in the bench scale experimental environment, and therefore in practice. The benefit of this two method design approach is that the modelled bulk behaviour is directly related to the physical material properties obtained via the standard bulk strength and friction assessment tests. To further aid the design process, it is then also possible to compare test, equipment or entire system performance through application of engineering mechanics (continuum approach) using physical properties with that simulated using virtual particle parameters. Feedback from validation tests allows continuous improvement of the methods applied. The general procedure is summarised in Figure 4.

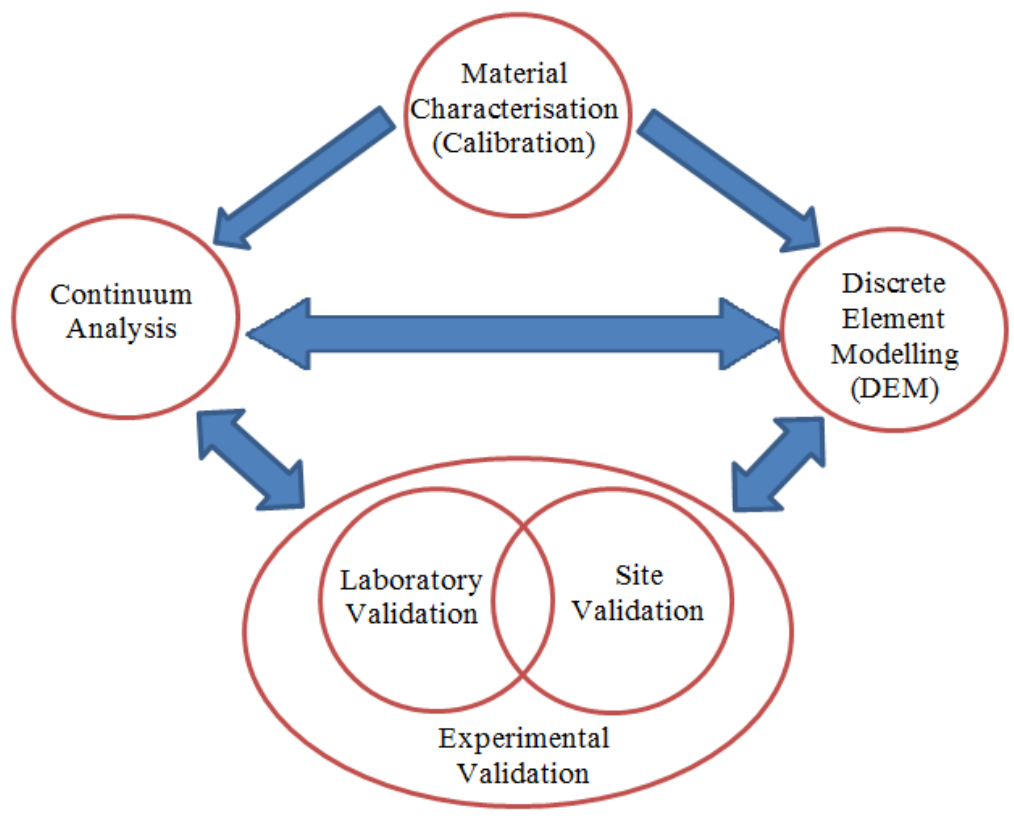

Figure 4: Complete Design Methodology [Ilic]

The similarities associated with scaling up the test equipment (to handle full size product and reflect physical material behaviour) and the challenge of characterising biomass materials become promptly apparent. This is further complicated by the elastic, fibrous and/or stringy nature of some feedstocks, where additional simulation parameters, related to individual particle (fibre) characteristics will require modification and adaptation of existing contact models and introduction of elasticity.

Currently, it is possible to either input or approximate the actual 3D shape of particles modelled into DEM [Williams et al]. Image analysis, particle 3D scanning or shape characterisation is performed as part of calibration test work. Particle shape characterisation, as well as size distribution analysis, has been implemented to qualitatively assess both segregation and particle attrition/degradation. Through accurate calibration and analysis, it is possible to optimise a system through modelling for minimised degradation or particle breakage [Donohue]. The calibration process involves quantifying the propensity of a representative particle (taken from a representative sample) to break, and once the particle does break, defining the resulting representative particle size distribution. This data is then input into the modelling package. Challenges are obviously faced when dealing with springy, elastic particles with either elastic or plastic deformation. Heat build-up due to handling biomass pellets through extracting numerical impact/shear density data (related to the relative velocity and applied load in each direction) to heat generated on a surface has also been investigated recently [llic]. This work formed part of the biomass transfer design at Drax power station in the U.K. 
Additionally, work has also been undertaken in refining existing theory to assess the mechanisms associated with densifying screw feeders, [Roberts et al]. In such systems, the aim is to achieve higher packing densities, to deaerate or dewater the bulk solid handled.

\section{Supply Chain Realities}

Inherently, there are a number of realities associated with the challenge of sourcing, transporting, handling and processing feedstocks along the handling or supply route from the source (producer) to refinery or conversion technology. Three major challenges are a) minimising contamination of the feedstock (soil or farming machinery parts for example), b) ensuring efficiency and operability for the range of heterogeneous nature of the feedstock handled and c) minimising the variability (degradation or friability) of the product during handling. These challenges are obstacles that are or will be encountered as biomass use in the renewable energy and fuel mix increases and becomes more prominent in the transition from a pilot to commercial scale (or pioneering) facility. If not controlled, such challenges will drive unexpected equipment problems that include high wear, clogging and failure of equipment, as well as secondary order effects such as dust generation and safety risks used in the handling and transportation process. For trade of such commodities, in addition to calorific value or ash content (typical utilisation properties) quality control of the feedstock, contamination free and product characteristics would be established through product specification criteria. The product specification criteria needs to be defined via a characterisation programme. As an example, Figure 4 below shows two different forms of sugarcane trash a) sourced and $b$ ) reduced with a mulcher $(45 \mathrm{~mm}$ throat size). Also shown in Figure 4 , is the heterogeneous nature of the sourced product showing the dried soil in mulched product $b$ ) and the contamination found in the sourced product $c$ ).

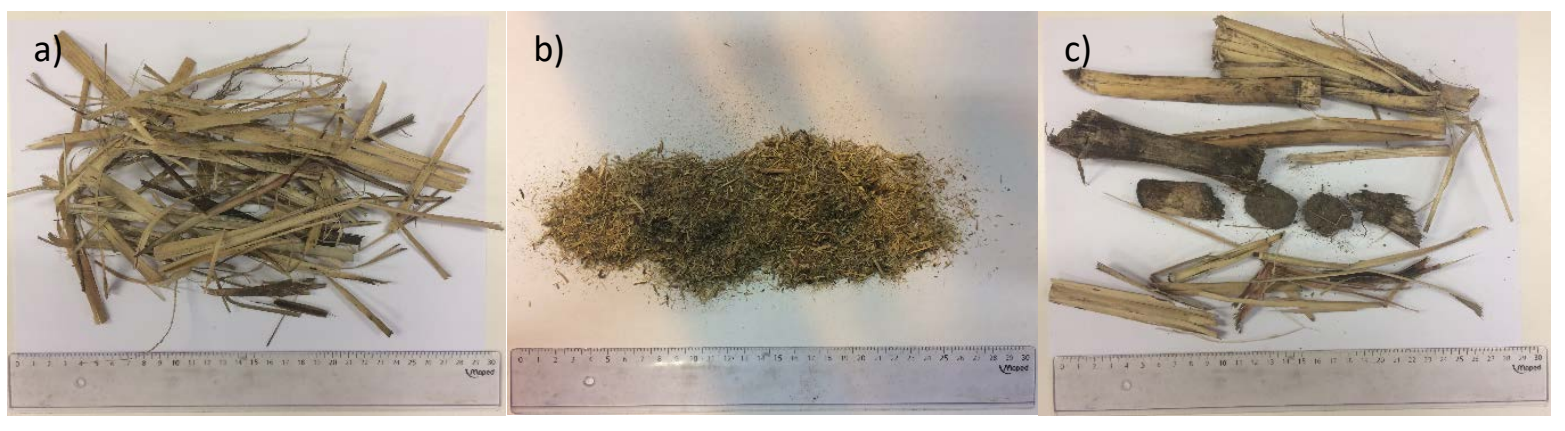

Figure 5: Variability of Sugarcane Trash

For a cellulosic bioethanol plant being economically successful and physically operatable, obtaining a low cost, low moisture, and low contamination biomass is essential. The cost of the biomass feedstock includes the purchase price from farmers, the transportation cost from fields to a plant, and the storage cost. Since the purchase price is more or less fixed or negotiated up front, the only option to lower the cost is to work on both the transportation logistics and storage strategy. To minimize transportation cost, typically a plant is built next to the feed source, for example, a 30 million gallons per year cornstover bioethanol plant may annually use 375,000 dry tons of cornstover, which can be provided by local farmers within a 30-mile radius of the facility. A sugar cane bagasse bioethanol plant has to be constructed next to the sugar plant and the available bagasse from the sugar plant will dictate the size of the cellulosic bioethanol plant.

The big challenges for transporting and storing agricultural crop residues or energy crops, such as cornstover, wheat straw, rice straw, switchgrass, and sorghum, are their high moisture content and low bulk density. After being harvested, the typical agricultural crop residues show a moisture as high as $60 \%$. High moisture means not only the wasted energy of hauling water by a truck trailer, but also 
the increasing risk of self-heating and self-degrading of the biomass during storage. The low bulk density will limit the carrying weight that the truck trailer can haul. That's one of the reasons that a rectangular bale is better than a round bale since rectangular bales haves a higher densification density than that of round bales.

Typically crop residues or energy crops are available only at one time of the year, while the energy demand or plant operation is likely to be all the year around. Storage is essential to ensure a smooth year round plant operation. The added storage cost includes the operational costs of moving biomass into and out of the store, and the capital invested in the storage facility. Low bulk density of the crop related biomass indicates large storage space is necessary. To minimize storage cost, biomass bales can be stored in an open field and covered with tarps to avoid water damage from either rain or snow. However, lighting strike pose a potential fire risk. For instance, in August 17, 2016, a suspected lightning strike set fire to a cornstover bale at a DuPont storage site located in Story County, lowa. The fire destroyed more than 10,000 bales - roughly 5,000 tons - of DuPont's cornstover repository supporting its Nevada, lowa cellulosic ethanol operation.

All these factors combine to ensure that agricultural biomass arrive at the plant site at a significant cost, the level of which can be a decisive factor in determining whether the cellulosic ethanol can compete with fossil fuels either at the current price, or at a projected future price.

How to minimize soil contamination in the collected crop residues or the energy crops is another important topic. One way to minimize the soil pickup is to select the right harvesting method. Any baling method will pick up soil from the field. For instance, the ash content in a standing cornstover, is about $3.5 \%$ of dry matter (DM), while the baled cornstover typically have an ash content around $8 \%$ when using rake or stalk chopper for windrowing. In general, over $60 \%$ of the ash is silicon dioxide, which can do significant damage, typically erosion to many types of processing equipment, such as grinding mills, pneumatic conveying pipes, rotary valves, agitators and pumps. Furthermore, inert material through the system does not produce ethanol at all. It is found that the performance of nonsoil removed cornstover can be $10 \%-15 \%$ lower than soil removed ones. Therefore, considering the poor handleability of biomass mentioned thus far, it presents a challenge job to design an efficient soil removal system.

\section{Identification of Key Design Parameters}

The envisioned physical properties, presenting themselves as potential barriers for adequate unification of design criteria for biomass feedstocks, are outlined below. They are drawn from a combination of previous studies undertaken in this area and practical application of solids design criteria to the traditional bulk solid commodities in the entire materials handling chain.

Critical Opening Dimension: In the traditional mined material resources sector, typical bulk solid materials guidelines for favourable design indicate that a ratio of 3:1 (plane flow) and 5:1 (conical) of characterising maximum particle size to minimum outlet opening dimension must be met to prevent mechanical arching and allow flow. Research such as a large-scale tester for evaluating mechanical arching effects [Hinterreiter] suggested image analysis to quantify particle shape as a means of assessing bridging. In this case, parameters from image analysis (shape factor and aspect ratio) could be used to explain unfavourable flow or bridging effects. The opening dimension is also related to the internal strength of the bulk solid handled, which in turn is dependent on the fines content, mineralogy and the moisture content. The typical means of establishing this characterising parameter is using a direct shear tester like Jenike shear, ring shear (e.g. Shulze tester). 
Storage: In addition to flow/no flow criteria discussed previously, another important consideration in continuous feedstock supply is storage. Storage space required will likely be significant footprint due to low biomass bulk density, the cyclic nature of the biomass collection and the need for continuous feed to the biomass process. Furthermore, and just as important, is the storage duration. Traditionally, commodities in the minerals and resources sector impact storage design through internal strength characteristics and with storage time, strength could increase, thereby requiring larger opening dimensions. Conversely, the storability of certain biomass feedstocks may be lengthy without problems (such as many dry lignocellulosic materials), however, other feedstocks with high water content are prone to decay, as well as safety hazards due to micro/biological reactions such as mould and spores [ref Khan]. Storage for prolonged periods also presents fire risks as will be discussed later in the document.

Bulk Compressibility and Dilation ("Springiness"): Typically compressibility testing is assessed in a chamber of known volume with the application of an increasing external load. For biomass feedstocks, it is envisaged that the dilation characteristics, due to a diminishing compressibility and into the tensile load rather than compressive, will also be of significance. Such characteristics will influence the general size of storage and handling facilities such as sheds, stockpiles, bins, conveyor width or cross section required and also influence selection of most appropriate type of storage, handling and transportation equipment. The design example presented in Figure 1 to Figure 3 illustrates the type of data that may be obtained from such an analysis.

Particle or Fibre Shape and Characteristics: No doubt that particle shape and individual particle or fibre characteristics are of influence on feedstock behaviour, including bulk density and the opening dimension required to prevent mechanical arching. Individual particle or fibre characteristics likely to influence handleability are also important in establishing new or modified contact models for use with numerical modelling. Traditionally, particle size distribution (PSD) analysis has been conducted by either dry or wet sieving. This obviously needs to be undertaken in view of stringy, fibrous materials and size and shape characterisation will more than likely involve image analysis (recognition) and latest technologies enabling it such as 3D scanning. It has been reported [Lam] that traditional sieve analysis cannot accurately represent the complexity associate with biomass feedstocks. Recent research has also identified the use of digital imaging techniques to adequately capture the particle characteristics in multiple dimensions.

Moisture Effects: Potential safety hazards due to biological processes such as mould or spores [Khan] are also associated with handling biomass feedstocks with an elevated moisture content. Furthermore, moisture allows for development of microorganisms that could also lead to decay. From a storage and handleability perspective, tests to determine handling of biomass feedstocks at elevated moisture content may also be required. Such distinction will be driven by criteria to effectively define the type of conveying and/or feeding which should be applied such as hydraulic, pneumatic or mechanical conveying.

Degradation Characteristics: Such characteristics have demanded research [Kotzur et al], [Wu et al] in areas driven by government incentives for use of biomass feedstock in pelletised form such as the U.K. This entails assessment of particle sizing (i.e. pellet length) before and after impact and has been assessed via drop tests and centrifugal testers. Transporting and/or conveying feedstocks over increased heights and at high speeds and throughputs exposes them to high impact velocities. Degradation characteristics are important to ascertain fines generation and ultimately expulsion of these particles leads to dust, which in turn can pose explosion and environmental issues. 
External Behaviour or Self-heating Characteristics: While it is known that biomass is used to generate a source of energy, moving (through transportation and conveying) the feedstock also exposes equipment and areas within the handling corridor to generation of heat and potential issues associated with high wear. Such effects have been reported in the Drax power plant in the UK during the handling of wood pellets and sunflower seeds at high throughputs and through significant fall heights. In the Drax power station handling system, the feedstocks were conveyed at very high throughputs $(2800 \mathrm{t} / \mathrm{h}$ ) and very significant fall heights (in excess of 15.0 to $20.0 \mathrm{~m}$ ). Furthermore, during storage, some biomass feedstocks are also prone to self-heating due to microbial effects and/or interaction with oxygen.

In addition, it is also clear that effective sampling criteria is also required so as to define a means of establishing representative samples that, when tested, will produce repeatable and reliable results. In determining the methodology to be used in the design process, it is paramount that the characteristics of biomass materials handled is well understood. Finally, the above parameters are also effectively defined by the characteristics required by the conversion process. A better understanding of handling parameters will also introduce potential for re-definition of the feedstock characteristics to be used in the conversion process.

\section{Fire and Safety Risks}

Some of the very properties that make cellulosic biomass an attractive fuel also make it a risky industrial feedstock. Risks encountered in handling and processing biomass include fire, dust inhalation, and toxic emissions. Failure to incorporate adequate safeguards when designing facilities to handle and process biomass can lead to accidents, higher-than-expected operational costs, expensive insurance premiums, and poor community relations.

In processing biomass for conversion to fuel or power, sparks can be generated during operations such as milling, grinding, and drying. For example, in a grinder, rocks entrained in raw feedstocks can cause sparks due to friction with blades which can ignite the feedstock itself or the resulting dust. Recent experiments have shown that biomass fires grow more quickly and spread more easily than many materials handled in industrial settings. Furthermore, combustible dusts, defined by OSHA as fine particles suspended in air that can present an explosion hazard, are produced during biomass handling and grinding operations. This dust can remain suspended in air, settle onto surfaces, or become trapped in storage bins. If a spark is generated, the dust presents a dangerous explosion hazard. Some biomass feedstocks, such as wood and grass pellets, also generate toxic gaseous emissions such as carbon monoxide, carbon dioxide, and methane. Build up of these gases, such as in closed storage containers, can be deadly. As biomass industries grow and mature, so too will the need for codes and standards to address the hazards associated with processing, handling, and storing biomass. For example, recent work to develop the ISO/TC238 solid biofuels standard included provisions on the prevention, detection, suppression and management of fires and explosions, as well as safe handling and storage, analysis of spontaneous heat generation and analysis of off-gassing products.

The emerging cellulosic biofuels industry, recognizing the need to better characterize the fire risk of biomass feedstocks, formed the Biomass Industry Panel on Codes and Standards in 2014. The Panel, sponsored by the U.S. Department of Energy Bioenergy Technologies Office, was formed in 2014 to improve codes/standards to better reflect current knowledge of biomass fire risk and industry practices. They are particularly focused on herbaceous feedstocks that have traditionally been handled predominantly on farms and outdoors but are now being produced as an industrial feedstock. To date, the BIPCS has submitted seven successful proposals to the International Code Council (ICC). These proposals aimed to a) clarify that biomass is NOT a hazardous material, b) allow for large 
biomass stacks and piles, c) add bioenergy feedstocks as a use to chapters focused on woody material, and d) add biomass categories for sprinkler design. A proposal is currently being developed to add corn stover and switchgrass bales to the commodity classification tables of the NFPA 13 Sprinkler Discharge Standard. Classification of stover and switchgrass bales will be based on experiments performed at UL to determine sprinkler discharge rates required to suppress an indoor bale fire. Insights gained from these experiments include the importance of bale density, bale shape, and feedstock type. The BIPCS is currently working with the International Code Council to prepare a technical document outlining ICC codes relevant to biomass-handling facilities. This document, targeted for release in early 2018, will assist engineers and reviewers in applying ICC codes for biomass-handling facilities. Future work also includes R\&D to better understand behavior of fires in herbaceous bale storage and develop best management practices to reduce the risk of fire in outdoor industrial storage yards.

Fire is another risk facing bioenergy feedstock supplies; it may not only cause feedstock shortages, but, more importantly, can inflict harm on people and property at the biorefinery or in the surrounding community. The current strategies for minimizing fire risk include spacing biomass stacks and piles far from other structures to reduce the likelihood of fire spread, and securing the area to minimize arson, a leading cause of biomass fires. Research to better understand fire behaviour in biomass storage stacks will lead to advanced storage systems - such as high-moisture storage-and biomass formats that reduce the risk of fire spread and minimize the threat of harm to people and property. The threat of fire can never be fully eliminated; rather, efforts to improve storage and handling design should concentrate on minimizing fire spread. Feedstock shortages due to fire can be reduced in the same manner as are other feedstock shortages-by improving access to feedstocks from a broader supply area.

An additional consideration regarding health aspects is the potential requirement for re-definition of tolerable particulate matter (PM) or total suspended particles (TSP) concentration when handling degradable fibrous or pelletised materials. As mentioned previously, prolonged storage and moisture effects can lead to generation of biological effects detrimental to health and a danger to personal safety. Both of these considerations add a new element requiring appraisal. Ultimately such matters may necessitate a re-definition of the Personal Protective Equipment (PPE) to be used by site personnel involved in the storage, handling and transportation of biomass feedstocks.

\section{Conclusions and Call for Additional Research}

The work embodied in this paper has presented an overview of the challenges facing the handling of solid biomass feedstocks from the perspectives of fundamental research, industry demand and practical application. These perspectives are driven by a unified objective to improve sustainability benchmarks and reduce detrimental environmental and societal impacts through consequences associated with meeting global energy and fuel requirements. Physical characteristics and their application have been identified as, arguably, the most overlooked challenge, but one of utmost significance to biomass competing as a valuable resource commodity and underpinning essential economic modelling of the supporting supply/logistic chains.

Barriers to define appropriate selection criteria for equipment and accompanying processes through accurate technical specification for handling, storage and transportation of biomass has been discussed. A brief example demonstrating the compaction/dilation response has been provided to supplement that discussion. Also presented is an outline of the threat of fire and occupational safety risk that handling, storing and transporting such commodities in increased throughputs poses. 
Obtaining an accurate representative sample has been discussed as the first step in effective biomass characterisation. In order to determine a selection technique for obtaining a representative sample, the most appropriate sampling test and procedure needs to be established for a range of feedstocks. This also undoubtedly requires categorisation of feedstock types into sub-groups based on a set of pre-defined physical characteristics. Benchmarking against established standardised tests and procedures, their application and limitation in the characterisation of biomass feedstocks is also required. This may involve classification related to particle shape, size, density, porosity, moisture, and biological behaviour, propensity for self-heating and degradation characteristics. It is important to note that as a traded commodity, the underlying biomass feedstock property specification is also heavily dictated by the optimum, most economically viable, conversion process or technology.

Investigation into the development of new test apparatus', methods and practices for determination of key handling properties such as elasticity, cyclic compressibility and dilation response, and friability or degradation need to be further evaluated. A comprehensive testing regime, encompassing a broad range of feedstocks and response to consolidation is required in which the influence of apparatus, sample volume and properties at the particle (or fibre) level are further investigated and the hysteretic compression/dilation relationship presented previously in Section 2 validated.

From an alternative point of view, some of the existing tests and procedures in use for calibrating numerical modelling (DEM) parameters appear directly translatable to characterise the physical properties of biomass feedstocks. Image characterisation and 3D scanning techniques, for particle size and shape evaluation currently in use to define virtual particles have already started to be employed for specification of physical feedstock properties. While simulation tools such as DEM provide benefits, they also pose limits that need to be further explored.

The greatest benefit of the characterisation approach described will be the ability to relate physical handleability properties to the energy, calorific and/or yield value of the feedstock. This will provide a much more complete and accurate set of design, operational and economic criteria to consider when deciding on the supply, logistics network, business model and ultimately, will contribute to valorisation of the biomass feedstock.

\section{References}

1. BP Statistical Review of World Energy, June 2016

2. U.S. Energy information Administration, International Energy Outlook 2016

3. Ramirez-Gomez, Research Needs on biomass Characterization to Prevent Handling Problems and Hazards in Industry

4. Sharma, Ingalls, Jones, Khanchi - Biomass Supply Chain Design and Analysis: Basis, Overview, Modeling, Challenges, and Future

5. Wu, Schott, Lodewijks - Physical Properties of Solid Biomass

6. Khan, Bradley, Berry and Reed - Best Practice Guide for Handling of Coal/Biomass Blends for CoFiring in Coal Power Plants

7. Kotzur, Berry, Bradley, Dias and e Silva - Influence of Pellet Length on Breakage by Impact

8. Ilic, PhD, 2013

9. Ilic, Continuum and DEM analysis in Transfer Chutes - MHEA

10. Johnstone - Calibration of DEM models for Granular Materials using Bulk Physical Tests

11. Groger, Katterfeld - Application of DEM in Materials Handling - Basics and Calibration

12. Grima, Wypych - Development and Validation of Calibration Methods for DEM

13. Roberts, Donohue, Ilic, Wakeham - An Investigation into the Performance of Screw Feeders used in Bulk Solid Densification Processes 
14. Hinterreiter, Hartmann, Turowski - Method for Determining Bridging properties of Biomass Fuels - Experimental and Model Approach

15. Donohue, Modelling Particle Breakage in Transfer Chutes with DEM

16. Miccio, Barletta, Poletto, Flow Properties and Arching Behaviour of Biomass Particulate Solids

17. Jenike, Gravity Flow of Bulk Solids, Bulletin 108

18. IRENA - Global bioenergy Supply and demand projections, Remap 2030

19. Shah, Darr, A techno-economic analysis of the corn stover feedstock supply system for cellulosic biorefineries

20. Kaliyan, Morey - Factor affecting strength and durability of densified biomass products

21. Craven, Swithenbank, Sharifi - Investigation into the Flow Properties of Coarse Solid Fuels for Use in Industrial Feed Systems

22. Lam, Sokhansanj - Engineering Properties of Biomass, Chapter 2, Engineering and Science of Biomass Feedstock Production and Provision

23. Williams, Chen, Weeger, Donohue, Particle shape characterisation and its application to discrete element modelling, Particuology 